\title{
Costo de la atención de la hipertensión arterial y su impacto en el presupuesto destinado a la salud en México
}

\author{
Enrique Villarreal-Ríos, MC, M en CSS, ${ }^{(1)}$ Alvaro Mathew-Q uiroz, MC, ${ }^{(2)}$ \\ María Eugenia $G$ arza-Elizondo, LE, $M$ en $C F_{1}^{(3)}$ Georgina N úñez-Rocha, MC, MSP, \\ A na María Salinas-Martínez, MC, D ra en SP, ${ }^{(3)}$ Martha Gallegos-H andal, MC. ${ }^{(4)}$
}

\section{Villarreal-Ríos E, Mathew-Quiroz A, Garza-Elizondo ME, Núñez-Rocha G, Salinas-Martínez AM, Gallegos-Handal M. Costo de la atención de la hipertensión arterial y su impacto en el presupuesto destinado a la salud en México \\ Salud Publica Mex 2002;44:7-13. El texto completo en inglés de este artículo está disponible en: http://www.insp.mx/salud/index.html}

\section{Resumen}

Objetivo. Determinar el costo de la atención de la hipertensión arterial, su impacto en el gasto en salud y en el producto interno bruto (PIB) de México. Material y métodos. Diseño ecológico, efectuado de junio a noviembre de 1999 en el Instituto Mexicano del Seguro Social (IMSS), en la ciudad de Monterrey, N uevo León, México. Se seleccionó aleatoriamente una muestra de expedientes clínicos con diagnóstico de hipertensión arterial, a los cuales se les contabilizó el uso de los servicios de salud y paralelamente se estimó el costo unitario por servicio. El costo por servicio y por paciente hipertenso se calculó ajustando el costo unitario en función de la utilización promedio y de un escenario extremo, cantidad que se proyectó a la población total de hipertensos y el resultado se comparó con el gasto anual en salud realizado en México. Resultados El costo anual por hipertenso en la Seguridad Social correspondió a \$1 067 en el escenario promedio y de $\$ 3913$ en el escenario extremo. El gasto anual en hipertensión arterial equivale a $13.95 \%$ del presupuesto destinado a la salud y $0.71 \%$ del PIB, valores que se modifican a $51.17 \%$ y $2.62 \%$ respectivamente en el escenario extremo. Conclusiones. La hipertensión arterial consume gran cantidad de recursos, por lo que deberá ser objeto de estudio
Villarreal-Ríos E, Mathew-Quiroz A,

Garza-Elizondo ME, Núñez-Rocha G,

Salinas-Martínez AM, Gallegos-Handal M.

The medical care costs of hypertension

and their impact on health

care resources in Mexico.

Salud Publica Mex 2002;44:7-13.

The English version of this paper

is available at: http://www.insp.mx/salud/index.html

\begin{abstract}
Objective. To assess the medical care costs of hypertension and their impact on the health care expenditures and on Mexico's G ross N ational Product (GN P). Material and Methods. An ecological study was conducted from June to N ovember 1999, at Instituto Mexicano del Seguro Social (Mexican Institute of Social Security, IMSS), in Monterrey, $\mathrm{N}$ uevo Leon, Mexico.A random sample of medical charts of patients with hypertension was selected, to extract data on utilization of health services and unitary costs per care episode. The cost per care episode and per hypertensive patient was calculated by adjusting the unitary cost as a function of standard and extreme utilization of IMSS health services. The resulting figure was then projected to the total population of hypertensive patients and compared to the annual health care expenditures of Mexico. Results The annual cost per patient with hypertension was $\$ 1,067$ in the standard scenario and $\$ 3,913$ in the extreme scenario. The annual expenditures from hypertension corresponded to $13.95 \%$ of the budget allocated to health care and to $0.71 \%$, of Mexico's G N P.T hese figures changed to $51.17 \%$ and $2.61 \%$ in the extreme scenario, respectively. Conclusions. The costs of hypertension medical care account for a good por-
\end{abstract}

(1) Unidad de Investigación Epidemiológica y en Servicios de Salud, Q uerétaro. Instituto Mexicano del Seguro Social, Q uerétaro, Q uerétaro de Arteaga, México.

(2) Hospital General de Zona No 17, Instituto Mexicano del Seguro Social, Monterrey N uevo León, México.

(3) Unidad de Investigación Epidemiológica y en Servicios de Salud. Instituto Mexicano del Seguro Social, Monterrey, N uevo León, México.

(4) Hospital General de Zona N 0. 4, Instituto Mexicano del Seguro Social, G uadalupe, Monterrey, N uevo León, México.

Fecha de recibido: 2 de junio de 2000 - Fecha de aprobado: 10 de octubre de 2001

Solicitud de sobretiros: Dr. Enrique Villarreal Ríos, Morelos 133 oriente, Condominio Monterrey, 5 o Piso, colonia Centro, CP 64000. Monterrey Nuevo León, México.

Correo electrónico:felibree@ infosel.net.mx. 
por equipos multidisciplinarios de salud, en la búsqueda de alternativas de manejo más eficientes. El texto completo en inglés de este artículo está disponible en:http://www.insp.mx/ salud/index.html

Palabras clave: costo; hipertensión; utilización, México tion of healthcare resources. This problem should be analyzed by multidisciplinary health teams in search of more efficient medical care alternatives. The English version of this paper is available at:http://w ww.insp.mx/salud/index.html

Key words: cost; hypertension; utilization; Mexico
L os sistemas de salud, al igual que el resto de la sociedad, actualmente se encuentran inmersos en la dinámica de la economía de la salud, por lo tanto la escasez de los recursos, el alto costo de la atención y el presupuesto asignado a la salud adquieren relevancia.

Por otro lado, la transición epidemiológica y el incremento en la esperanza de vida han influido en la aparición de padecimientos crónico degenerativos altamente demandantes de servicios de salud. De estos padecimientos, al ocupar los primeros lugares de atención, destacan los cardiovasculares; entre ellos, la hipertensión arterial reporta aproximadamente 50 millones de casos en Estados Unidos de América, ${ }^{1}$ en tanto en Canadá y México la prevalencia alcanza $25^{2}$ y $26.6 \%,{ }^{3}$ respectivamente.

En este último país, en 1998, se reportaron 481368 casos nuevos y durante 1999 la cifra correspondió a 321 387. ${ }^{4}$ Esto sin considerar que por cada hipertenso diagnosticado se estima que existen 1.3 que no lo han sido. ${ }^{3}$

Se ha descrito en la literatura que el costo anual de la atención del paciente hipertenso ambulatorio varía entre 57 y 450 dólares, ${ }^{5}$ incluyendo pruebas de laboratorio y medicamento.

Lo anterior señala la magnitud del padecimiento y su impacto en la sociedad, no sólo en el área de la epidemiología y la salud pública, sino en el aspecto económico, y por ello la importancia del uso racional de los recursos en el sector salud.

Ubicados en este contexto, el objetivo del presente trabajo fue determinar el costo de la atención de la hipertensión arterial, su impacto en el gasto en salud y en el producto interno bruto (PIB) de México.

\section{Material y métodos}

Se trata de un estudio realizado de junio a noviembre de 1999. La metodología incluyó cuatro grandes apartados: intensidad de uso de los servicios de salud, estimación de los costos unitarios, definición de la población de hipertensos y la identificación del gasto en salud. Para cada uno se empleó un abordaje específico acompañado de supuestos, los cuales se señalan en cada sección.

El tipo y la intensidad de uso de los servicios de salud fueron identificados mediante la información con- tenida en el expediente clínico. Estos fueron seleccionados aleatoriamente de cinco Unidades de Medicina Familiar del área urbana, estratificando por consultorio médico y turno (matutino y vespertino). De esta población se analizaron también aquellos que además habían utilizado el servicio de segundo y tercer nivel de atención. Como criterio de inclusión se consideraron los expedientes con diagnóstico de hipertensión arterial de más de un año de evolución, y se analizó el comportamiento en el uso de los servicios de salud a lo largo de 12 meses.

El tamaño de la muestra ( $\mathrm{N}=214)$ fue establecido utilizando la fórmula de proporciones para población infinita, considerando que $15 \%$ o más de la población general es hipertensa: $p=0.15 ; Z=1.64 ; \mathrm{d}=0.04$.

Las variables incluidas en la encuesta fueron el tipo y la intensidad de utilización de los servicios de salud (consulta externa, medicamentos, hospitalización, laboratorio, radiología y algunos otros procedimientos especializados). De cada una de ellas se obtuvieron promedios y la desviación estándar.

Paralelamente se estimaron los costos de la atención médica. Para ello se eligieron una Unidad de Medicina Familiar y un Hospital General de Zona pertenecientes al IMSS; en cada uno de ellos se estimaron los costos unitarios de atención, utilizando el modelo de presupuesto capitado. ${ }^{6}$

Para la estimación de los costos fijos unitarios, este modelo identifica servicios generales y servicios finales; considera como insumos de cada uno de los servicios incluidos los siguientes: recursos materiales (edificio y terreno), recursos físicos (mobiliario equipo e instrumental), recursos humanos (directivos, secretarias, médicos, enfermeras y personal auxiliar), servicios básicos (electricidad, agua, gas, teléfono) y material de oficina.

El gasto en servicios generales es desagregado e imputado a servicios finales de acuerdo con ponderadores dinámicos construidos ex profeso; este gasto, más el propio del servicio final, fueron ajustados por la productividad del servicio analizado para así definir el costo unitario fijo de cada uno de los servicios finales.

El costo variable unitario de cada uno de los motivos de demanda de servicio se construyó identificando consumos promedio de insumos (medicamentos y ma- 
terial de curación) para un caso típico; esto fue definido por grupos de expertos en el área.

La suma del costo fijo unitario y el costo variable unitario se convirtió en el costo unitario de cada uno de los motivos de demanda de servicio.

El costo estimado para las unidades médicas del IMSS se asumió como el costo para la población perteneciente a la Seguridad Social: Instituto Mexicano del Seguro Social (IMSS), Instituto de Seguridad y Servicios Sociales de los Trabajadores del Estado (ISSSTE), Petróleos Mexicanos (Pemex), Secretaría de Defensa Nacional (Sedena) y los Institutos Estatales de Salud con Población Cautiva (Servicios Estatales). El costo para la población abierta atendida por el Estado (Secretaría de Salud, Departamento del Distrito Federal e IMSSSolidaridad) se estimó aplicando como ponderador 0.66 . Este ponderador representa la proporción de costo de la consulta de la Secretaría de Salud (\$45), en relación con el costo de la consulta de Medicina Familiar en la Seguridad Social (\$68). Para la Medicina Privada se adoptó como ponderador 2.69, construido considerando la proporción del costo de la consulta privada (\$300) en relación con el costo de la consulta de medicina interna de la seguridad social (\$111). Estos ponderadores se aplicaron al costo de cada uno de los servicios incluidos en este estudio.

Para definir el total de hipertensos en el país se utilizaron los criterios de la Encuesta Nacional de Enfermedades Crónicas, ${ }^{3}$ en la cual se señala la prevalencia de hipertensión en mayores de 20 años en México. (Norte $27.9 \%$, Centro $26.9 \%$, Sur 27.8\% y Distrito Federal (DF) $23.8 \%$ ). Esta información se relacionó con el total de la población existente en la República Mexicana (94 962 160). ${ }^{7}$

Para definir la cantidad de población, por tipo de institución que proporciona la atención médica, se utilizó la información señalada en Estructura del mercado de los servicios de salud en México: $84 \%$ para la seguridad social, $27 \%$ para la población abierta atendida por el estado y $19 \%$ para la medicina privada.

Para la identificación del gasto en salud y el PIB de México se utilizó la información de las Cuentas Nacionales de Salud, ${ }^{9}$ correspondiendo a \$128 706 y \$2 523648 millones, respectivamente, para 1996.

Se construyeron dos escenarios de utilización de los servicios de salud; el primero, construido sobre la base del uso promedio; el segundo, catalogado como escenario extremo, se integró con la suma del promedio y la desviación estándar. En cada escenario se estimó el costo total por nivel de atención y por institución, ajustado por la cantidad de población atendida. La suma correspondiente representó el costo total de la atención de la hipertensión. Finalmente, se comparó con el gasto total destinado a la salud y con el PIB.

\section{Resultados}

La edad promedio de la población estudiada fue de 61.78 años, $\pm 10.90,63.72 \%$ refirieron la primaria como escolaridad máxima y predominó el sexo femenino (65.6\%). El tiempo promedio del primer registro en el expediente clínico fue de 7.02 años, \pm 4.12 y el promedio de las tres últimas cifras de tensión diastólica correspondió a $84.14 \mathrm{~mm} \mathrm{Hg}, \pm 6.67$.

Medicina Familiar fue el servicio más utilizado con 8.437 visitas al año en promedio por hipertenso, \pm 2.96 ; la glicemia, el estudio de laboratorio más utilizado en primer nivel de atención, tuvo un promedio de $1.16, \pm 1.69$; y se hicieron $0.372 \pm 0.53$ estudios radiológicos de tórax por hipertenso. En el cuadro I se muestra el uso promedio anual por servicio y el valor correspondiente al escenario extremo.

El promedio de visitas a cardiología fue de $0.172, \pm$ 0.59; la estancia hospitalaria promedio en segundo nivel de atención fue de $0.014 \pm 0.205$ días cama al año por hipertenso; en tanto, en el tercer nivel de atención fue de $0.163 \pm 1.194$. El resto de los servicios utilizados y el promedio de uso por hipertenso se muestra en los cuadros II y III. Aquí también se presenta el uso de los servicios en el escenario extremo.

El costo unitario más alto en la seguridad social fue el del cateterismo cardiaco, \$ 3 362; el día cama en cardiología (alta especialidad) y en el segundo nivel de atención correspondió a 1249 y 917 pesos respectivamente; en tanto el costo de la consulta externa de especialidad fue de 111 pesos. En los cuadros I, II y III se presenta el costo unitario del resto de los servicios utilizados, así como los estimados para las Instituciones del Estado y Medicina Privada.

En el escenario promedio, el servicio más costoso por hipertenso por año fue el de medicina familiar con 1554 pesos, seguido por el día cama en cardiología (alta especialidad), de 547 pesos. Este último servicio también fue el más costoso en el escenario extremo; 4557 pesos. El costo anual para cada uno de los servicios por tipo de institución se muestra en los cuadros I, II y III.

En la seguridad social el costo anual de la atención del hipertenso en el escenario promedio, fue de 1067 pesos; en Instituciones del estado 701 y 2868 en la medicina privada. Estas cifras se modificaron en el escenario extremo a 3 913; 2570 y 10518 pesos, respectivamente. En el cuadro IV se presenta el costo por nivel de atención y escenario.

El costo total de la hipertensión en México para el escenario promedio correspondió a 17953 millones de pesos, de acuerdo con una población de hipertensos estimada en 13704573 (prevalencia de 1443 por cada 


\section{Cuadro I \\ Costo UNITARIO, UTILIZACIÓN DE LOS SERVICIOS Y COSTO ANUAL POR HIPERTENSO E INSTITUCIÓN, Según escenario en Primer Nivel de Atención. México, 1999}

\begin{tabular}{|c|c|c|c|c|c|c|c|c|c|c|c|c|c|}
\hline \multirow[b]{3}{*}{ Servicio } & \multirow{2}{*}{\multicolumn{3}{|c|}{ Costo unitario }} & \multirow[b]{3}{*}{$\begin{array}{c}\text { Unidad } \\
\text { de me- } \\
\text { dida }\end{array}$} & \multicolumn{5}{|c|}{ Escenario promedio } & \multicolumn{4}{|c|}{ Escenario extremo } \\
\hline & & & & & \multirow[b]{2}{*}{$\begin{array}{l}\text { Utiliza- } \\
\text { ción }\end{array}$} & \multirow[b]{2}{*}{$\begin{array}{l}\text { Desvia- } \\
\text { ción es- } \\
\text { tándar }\end{array}$} & \multicolumn{3}{|c|}{$\begin{array}{c}\text { Costo anual ajustado } \\
\text { por utilización }\end{array}$} & \multirow[b]{2}{*}{$\begin{array}{l}\text { Utiliza- } \\
\text { ción }\end{array}$} & \multicolumn{3}{|c|}{$\begin{array}{c}\text { Costo anual ajustado } \\
\text { por utilización }\end{array}$} \\
\hline & $\begin{array}{l}\text { Seguridad } \\
\text { social }\end{array}$ & $\begin{array}{l}\text { Secreta- } \\
\text { ría de } \\
\text { Salud }\end{array}$ & $\begin{array}{l}\text { Medi- } \\
\text { cina } \\
\text { privada }\end{array}$ & & & & $\begin{array}{l}\text { Seguri- } \\
\text { dad } \\
\text { social }\end{array}$ & $\begin{array}{l}\text { Secreta- } \\
\text { ría de } \\
\text { Salud }\end{array}$ & $\begin{array}{c}\text { M edi- } \\
\text { cina } \\
\text { privada }\end{array}$ & & $\begin{array}{l}\text { Seguri- } \\
\text { dad } \\
\text { social }\end{array}$ & $\begin{array}{l}\text { Secreta- } \\
\text { ría de } \\
\text { Salud }\end{array}$ & $\begin{array}{c}\text { Medi- } \\
\text { cina } \\
\text { privada }\end{array}$ \\
\hline Medicina familiar & 68.53 & 45.00 & 184.20 & Consulta & 8.437 & 2.969 & 578.15 & 379.64 & 1554.07 & 11.406 & 781.60 & 513.24 & 2100.95 \\
\hline Biometría hemática & 20.79 & 13.65 & 55.88 & Estudio & 0.563 & 0.739 & 11.70 & 7.69 & 31.46 & 1.302 & 27.07 & 17.77 & 72.76 \\
\hline Glicemia & 26.57 & 17.45 & 71.42 & Estudio & 1.167 & 1.694 & 31.01 & 20.36 & 83.35 & 2.861 & 76.02 & 49.92 & 204.33 \\
\hline Creatinina & 27.81 & 18.26 & 74.75 & Estudio & 0.288 & 0.564 & 8.01 & 5.26 & 21.53 & 0.852 & 23.69 & 15.56 & 63.69 \\
\hline Urea & 26.98 & 17.72 & 72.52 & Estudio & 0.242 & 0.536 & 6.53 & 4.29 & 17.55 & 0.778 & 20.99 & 13.78 & 56.42 \\
\hline Colesterol & 27.22 & 17.87 & 73.17 & Estudio & 0.609 & 0.846 & 16.58 & 10.89 & 44.56 & 1.455 & 39.61 & 26.01 & 106.46 \\
\hline Triglicéridos & 26.77 & 17.58 & 71.96 & Estudio & 0.028 & 0.289 & 0.75 & 0.49 & 2.01 & 0.317 & 8.49 & 5.57 & 22.81 \\
\hline General de orina & 22.38 & 14.70 & 60.16 & Estudio & 1.056 & 1.648 & 23.63 & 15.52 & 63.53 & 2.704 & 60.52 & 39.74 & 162.67 \\
\hline Acido úrico & 27.81 & 18.26 & 74.75 & Estudio & 0.298 & 0.645 & 8.29 & 5.44 & 22.28 & 0.943 & 26.22 & 17.22 & 70.49 \\
\hline Radiografía de tórax & 113.06 & 74.24 & 303.91 & Estudio & 0.372 & 0.539 & 42.06 & 27.62 & 113.05 & 0.911 & 103.00 & 67.63 & 276.86 \\
\hline Electrocardiograma & 20.00 & 13.13 & 53.76 & Estudio & 0.112 & 0.316 & 2.24 & 1.47 & 6.02 & 0.428 & 8.56 & 5.62 & 23.01 \\
\hline $\begin{array}{l}\text { Costo total } \\
\text { por hipertenso }\end{array}$ & 407.92 & 267.86 & 1096.48 & & & & 728.95 & 478.67 & 1959.41 & & 1175.77 & 772.06 & 3160.45 \\
\hline
\end{tabular}

\section{Cuadro II \\ Costo UNITARIO, UTILIZACIÓN DE LOS SERVICIOS Y COSTO ANUAL POR HIPERTENSO E INSTITUCIÓN, Según escenario en Segundo nivel de atención. México, 1999}

\begin{tabular}{|c|c|c|c|c|c|c|c|c|c|c|c|c|c|}
\hline \multirow[b]{2}{*}{ Servicio } & \multicolumn{3}{|c|}{ Costo unitario } & \multirow[b]{2}{*}{$\begin{array}{c}\text { Unidad } \\
\text { de me- } \\
\text { dida }\end{array}$} & & Esce & $\frac{\text { nario prol }}{\mathrm{Co}}$ & $\begin{array}{l}\text { dio } \\
\text { anual aju } \\
\text { or utilizaci }\end{array}$ & & & $\frac{\text { Escenari }}{\mathrm{Co}}$ & $\begin{array}{l}\text { extremo } \\
0 \text { anual aju } \\
\text { or utilizac }\end{array}$ & \\
\hline & $\begin{array}{l}\text { Seguridad } \\
\text { social }\end{array}$ & $\begin{array}{l}\text { Secreta- } \\
\text { ría de } \\
\text { Salud }\end{array}$ & $\begin{array}{c}\text { Medi- } \\
\text { cina } \\
\text { privada }\end{array}$ & & $\begin{array}{l}\text { Utiliza- } \\
\text { ción }\end{array}$ & $\begin{array}{l}\text { Desvia- } \\
\text { ción es- } \\
\text { tándar }\end{array}$ & $\begin{array}{l}\text { Seguri- } \\
\text { dad } \\
\text { social }\end{array}$ & $\begin{array}{l}\text { Secreta- } \\
\text { ría de } \\
\text { Salud }\end{array}$ & $\begin{array}{c}\text { Medi- } \\
\text { cina } \\
\text { privada }\end{array}$ & $\begin{array}{l}\text { Utiliza- } \\
\text { ción }\end{array}$ & $\begin{array}{l}\text { Seguri- } \\
\text { dad } \\
\text { social }\end{array}$ & $\begin{array}{l}\text { Secreta- } \\
\text { ría de } \\
\text { Salud }\end{array}$ & $\begin{array}{c}\text { Medi- } \\
\text { cina } \\
\text { privada }\end{array}$ \\
\hline Consulta & & & & & & & & & & & & & \\
\hline Medicina interna & 111.61 & 73.29 & 300.00 & Consulta & 0.153 & 0.634 & 17.08 & 11.21 & 45.90 & 0.787 & 87.83 & 57.68 & 236.10 \\
\hline $\begin{array}{l}\text { Consulta } \\
\text { cardiología }\end{array}$ & 111.61 & 73.29 & 300.00 & Consulta & 0.172 & 0.591 & 19.20 & 12.61 & 51.60 & 0.763 & 85.15 & 55.92 & 228.90 \\
\hline $\begin{array}{l}\text { Consulta de } \\
\text { urgencias }\end{array}$ & 44.6 & 29.29 & 119.88 & Consulta & 0.126 & 0.430 & 5.62 & 3.69 & 15.11 & 0.556 & 24.80 & 16.28 & 66.66 \\
\hline $\begin{array}{l}\text { Consulta } \\
\text { oftalmología }\end{array}$ & 66.93 & 43.95 & 179.91 & Consulta & 0.195 & 1.009 & 13.05 & 8.57 & 35.08 & 1.204 & 80.58 & 52.92 & 216.61 \\
\hline Biometría hemática & 20.79 & 13.65 & 55.88 & Estudio & 0.102 & 0.397 & 2.12 & 1.39 & 5.70 & 0.499 & 10.37 & 6.81 & 27.89 \\
\hline Glicemia & 26.57 & 17.45 & 71.42 & Estudio & 0.098 & 0.380 & 2.60 & 1.71 & 7.00 & 0.478 & 12.70 & 8.34 & 34.14 \\
\hline Creatinina & 27.81 & 18.26 & 74.75 & Estudio & 0.065 & 0.283 & 1.81 & 1.19 & 4.86 & 0.348 & 9.68 & 6.35 & 26.01 \\
\hline Urea & 26.98 & 17.72 & 72.52 & Estudio & 0.056 & 0.268 & 1.51 & 0.99 & 4.06 & 0.324 & 8.74 & 5.74 & 23.50 \\
\hline Ego & 22.38 & 14.70 & 60.16 & Estudio & 0.056 & 0.285 & 1.25 & 0.82 & 3.37 & 0.341 & 7.63 & 5.01 & 20.51 \\
\hline Colesterol & 27.22 & 17.87 & 73.17 & Estudio & 0.023 & 0.179 & 0.63 & 0.41 & 1.68 & 0.202 & 5.50 & 3.61 & 14.78 \\
\hline Amilasa & 26.57 & 17.45 & 71.42 & Estudio & 0.009 & 0.096 & 0.24 & 0.16 & 0.64 & 0.105 & 2.79 & 1.83 & 7.50 \\
\hline Enzimas cardiacas & 79.71 & 52.34 & 214.26 & Estudio & 0.060 & 0.275 & 4.78 & 3.14 & 12.86 & 0.335 & 26.70 & 17.53 & 71.78 \\
\hline Radiografía de tórax & 113.06 & 74.24 & 303.91 & Estudio & 0.112 & 0.344 & 12.66 & 8.31 & 34.04 & 0.456 & 51.56 & 33.85 & 138.58 \\
\hline $\begin{array}{l}\text { Radiografía } \\
\text { de abdomen }\end{array}$ & 113.06 & 74.24 & 303.91 & Estudio & 0.005 & 0.068 & 0.57 & 0.37 & 1.52 & 0.073 & 8.25 & 5.42 & 22.19 \\
\hline Ecografía & 113.06 & 74.24 & 303.91 & Estudio & 0.014 & 0.118 & 1.58 & 1.04 & 4.25 & 0.132 & 14.92 & 9.80 & 40.12 \\
\hline Tomografía & 121.00 & 79.45 & 325.25 & Estudio & 0.009 & 0.096 & 1.09 & 0.72 & 2.93 & 0.105 & 12.71 & 8.34 & 34.15 \\
\hline Electrocardiograma & 20.00 & 13.13 & 53.76 & Estudio & 0.256 & 0.592 & 5.12 & 3.36 & 13.76 & 0.848 & 16.96 & 11.14 & 45.59 \\
\hline Días hospitalización & 917.52 & 602.49 & $2,466.30$ & Día & 0.014 & 0.205 & 12.85 & 8.43 & 34.53 & 0.219 & 200.94 & 131.94 & 540.12 \\
\hline $\begin{array}{l}\text { Costo total } \\
\text { por hipertenso }\end{array}$ & 90.48 & 1307.05 & $5,350.41$ & & & & 103.76 & 68.12 & 278.89 & & 667.81 & 438.51 & 1795.13 \\
\hline
\end{tabular}




\section{Cuadro III \\ Costo UNITARIO, UTILIZACIÓN DE LOS SERVICIOS Y COSTO ANUAL POR HIPERTENSO E INSTITUCIÓN, SEgún esCenario en terCer nivel de ATEnCIÓn. MéXICO, 1999}

\begin{tabular}{|c|c|c|c|c|c|c|c|c|c|c|c|c|c|}
\hline \multirow[b]{3}{*}{ Servicio } & \multirow{2}{*}{\multicolumn{3}{|c|}{ Costo unitario }} & \multirow[b]{3}{*}{$\begin{array}{c}\text { Unidad } \\
\text { de me- } \\
\text { dida }\end{array}$} & \multicolumn{5}{|c|}{ Escenario promedio } & \multicolumn{4}{|c|}{ Escenario extremo } \\
\hline & & & & & \multirow[b]{2}{*}{$\begin{array}{l}\text { Utiliza- } \\
\text { ción }\end{array}$} & \multirow[b]{2}{*}{$\begin{array}{l}\text { Desvia- } \\
\text { ción es- } \\
\text { tándar }\end{array}$} & \multicolumn{3}{|c|}{$\begin{array}{l}\text { Costo anual ajustado } \\
\text { por utilización }\end{array}$} & \multirow[b]{2}{*}{$\begin{array}{l}\text { Utiliza- } \\
\text { ción }\end{array}$} & \multicolumn{3}{|c|}{$\begin{array}{c}\text { Costo anual ajustado } \\
\text { por utilización }\end{array}$} \\
\hline & $\begin{array}{l}\text { Seguridad } \\
\text { social }\end{array}$ & $\begin{array}{l}\text { decreta- } \\
\text { ría de } \\
\text { Salud }\end{array}$ & $\begin{array}{l}\text { Medi- } \\
\text { cina } \\
\text { privada }\end{array}$ & & & & $\begin{array}{l}\text { Seguri- } \\
\text { dad } \\
\text { social }\end{array}$ & $\begin{array}{l}\text { Secreta- } \\
\text { ría de } \\
\text { Salud }\end{array}$ & $\begin{array}{l}\text { Medi- } \\
\text { cina } \\
\text { privada }\end{array}$ & & $\begin{array}{l}\text { Seguri- } \\
\text { dad } \\
\text { social }\end{array}$ & $\begin{array}{l}\text { Secreta- } \\
\text { ría de } \\
\text { Salud }\end{array}$ & $\begin{array}{l}\text { Medi- } \\
\text { cina } \\
\text { privada }\end{array}$ \\
\hline Biometría hemática & 20.79 & 13.65 & 55.88 & Estudio & 0.042 & 0.325 & 0.87 & 0.57 & 2.35 & 0.367 & 7.63 & 5.01 & 20.51 \\
\hline Glicemia & 26.57 & 17.45 & 71.42 & Estudio & 0.042 & 0.310 & 1.12 & 0.73 & 3.00 & 0.352 & 9.35 & 6.14 & 25.14 \\
\hline Creatinina & 27.81 & 18.26 & 74.75 & Estudio & 0.037 & 0.271 & 1.03 & 0.68 & 2.77 & 0.308 & 8.57 & 5.62 & 23.02 \\
\hline Urea & 26.98 & 17.72 & 72.52 & Estudio & 0.037 & 0.271 & 1.00 & 0.66 & 2.68 & 0.308 & 8.31 & 5.46 & 22.34 \\
\hline General de orina & 22.38 & 14.70 & 60.16 & Estudio & 0.014 & 0.152 & 0.31 & 0.21 & 0.84 & 0.166 & 3.72 & 2.44 & 9.99 \\
\hline Colesterol & 27.22 & 17.87 & 73.17 & Estudio & 0.014 & 0.152 & 0.38 & 0.25 & 1.02 & 0.166 & 4.52 & 2.97 & 12.15 \\
\hline Triglicéridos & 26.77 & 17.58 & 71.96 & Estudio & 0.014 & 0.152 & 0.37 & 0.25 & 1.01 & 0.166 & 4.44 & 2.92 & 11.95 \\
\hline Enzimas & 79.71 & 52.34 & 214.26 & Estudio & 0.005 & 0.068 & 0.40 & 0.26 & 1.07 & 0.073 & 5.82 & 3.82 & 15.64 \\
\hline Radiografía de tórax & 110.00 & 72.23 & 295.68 & Estudio & 0.023 & 0.179 & 2.53 & 1.66 & 6.80 & 0.202 & 22.22 & 14.59 & 59.73 \\
\hline Ultrasonido & 113.06 & 74.24 & 303.91 & Estudio & 0.014 & 0.118 & 1.58 & 1.04 & 4.25 & 0.132 & 14.92 & 9.80 & 40.12 \\
\hline Tomografía & 212.00 & 139.21 & 569.86 & Estudio & 0.009 & 0.096 & 1.91 & 1.25 & 5.13 & 0.105 & 22.26 & 14.62 & 59.83 \\
\hline Electrocardiograma & 58.00 & 38.09 & 155.90 & Estudio & 0.037 & 0.253 & 2.15 & 1.41 & 5.77 & 0.290 & 16.82 & 11.04 & 45.21 \\
\hline Cateterismo & 3362.00 & 2207.65 & 9037.07 & Estudio & 0.005 & 0.068 & 16.81 & 11.04 & 45.19 & 0.073 & 245.43 & 161.16 & 659.71 \\
\hline Días hospital & 1249.44 & 820.44 & 3358.50 & Día & 0.163 & 1.194 & 203.66 & 133.73 & 547.44 & 1.357 & 1695.49 & 1113.34 & 4557.48 \\
\hline $\begin{array}{l}\text { Costo total } \\
\text { por hipertenso }\end{array}$ & 5362.73 & 3521.43 & 4415.04 & & & & 234.12 & 153.74 & 629.32 & & 2069.5 & 1358.93 & 5562.82 \\
\hline
\end{tabular}

\section{Cuadro IV \\ Costo ANUAL POR HIPERTENSO Y TOTAL POR POBLACIÓN DE HIPERTENSOS AJUSTADO POR TIPO DE INSTITUCIÓN, SEgún esCenario PROPUeSTO. México, 1999}

\begin{tabular}{|c|c|c|c|c|c|}
\hline \multirow[b]{2}{*}{ Tipo de institución } & \multirow[b]{2}{*}{$\begin{array}{l}\text { Población por } \\
\text { tipo de atención }\end{array}$} & \multicolumn{2}{|c|}{ Escenario promedio } & \multicolumn{2}{|c|}{ Escenario extremo } \\
\hline & & $\begin{array}{l}\text { Costo anual } \\
\text { por hipertenso }\end{array}$ & $\begin{array}{c}\text { Costo anual por población } \\
\text { de hipertensos }\end{array}$ & $\begin{array}{l}\text { Costo anual } \\
\text { por hipertenso }\end{array}$ & $\begin{array}{c}\text { Costo anual por } \\
\text { población de hipertensos }\end{array}$ \\
\hline \multicolumn{6}{|l|}{ 'imer nivel } \\
\hline Seguridad social & 7400469 * & 729 & 5394535377 & 1176 & 8701177678 \\
\hline Población abierta & 3700235 * & 479 & 1771151991 & 772 & 2856799909 \\
\hline Medicina privada & $2603869 *$ & 1959 & 5102038211 & 3160 & 8229391022 \\
\hline $\begin{array}{l}\text { Costo total } \\
\text { gundo nivel }\end{array}$ & & & 12267725580 & & 19787368610 \\
\hline Seguridad social & & 104 & 767812713 & 668 & 4942189042 \\
\hline Población abierta & & 68 & 252090851 & 439 & 1622636122 \\
\hline Medicina privada & & 279 & 726181131 & 1795 & 4674218553 \\
\hline $\begin{array}{l}\text { Costo total } \\
\text { rcer nivel }\end{array}$ & & & 1746084696 & & 11239043718 \\
\hline Seguridad social & & 234 & 1, 732594931 & 2069 & 15315225552 \\
\hline Población abierta & & 154 & 568851393 & 1359 & 5028346343 \\
\hline Medicina privada & & 629 & 1638651881 & 5563 & 14484818529 \\
\hline $\begin{array}{l}\text { Costo total } \\
\text { lobal }\end{array}$ & & & 3940098204 & & 34828390424 \\
\hline Seguridad social & & 1067 & 7894943021 & 3913 & 28958592273 \\
\hline Población abierta & & 701 & 2592094236 & 2570 & 9507782375 \\
\hline Medicina privada & & 2868 & 7466871223 & 10518 & 27388428104 \\
\hline Costo total & & & 17953908480 & & 65854802752 \\
\hline
\end{tabular}

Las cifras se expresan en pesos

* Corresponde al número de hipertensos por tipo de institución 
100 habitantes). Para el escenario extremo el costo se modificó a 65854 millones de pesos. En el cuadro V se muestra el porcentaje del gasto en salud y el correspondiente al PIB para los dos escenarios propuestos.

\section{Discusión}

La prevalencia de la hipertensión arterial en la población en general se incrementa con la edad; este patrón de comportamiento es similar al de la población estudiada. En relación con el sexo, se encontró un mayor porcentaje de usuarios de los servicios de salud pertenecientes al sexo femenino, aunque se sabe que para el caso de la hipertensión arterial, la prevalencia es mayor en el sexo masculino según lo señalado en la Encuesta Nacional de Enfermedades Crónicas (ENEC). ${ }^{3}$

Una limitante para conocer el tiempo real de evolución de la hipertensión en la población estudiada fue la inconsistencia de esta variable en el expediente clínico; en función de ello se adoptó la fecha del primer registro de atención médica otorgada por este motivo. En relación con esto es oportuno comentar que el tiempo de evolución y el tiempo en control no necesariamente coinciden, pero también es una realidad que el paciente hipertenso, en la mayoría de las ocasiones, se entera de su padecimiento al recibir atención médica. Esto ha sido descrito en la ENEC, la cual reporta que por cada hipertenso diagnosticado existen 1.3 que no han sido identificados como tales. ${ }^{3}$

Es importante resaltar que el promedio del registro de las tres últimas cifras de la tensión arterial diastólica se ubica dentro de parámetros normales y cataloga a esta población como controlada, lo cual, suponemos, se relaciona estrechamente con el uso de los servicios de salud.

Como era de esperarse, el servicio más utilizado correspondió al de medicina familiar, situación lógica si se considera a éste como el responsable del control primario de la población hipertensa. Aunque es conveniente aclarar que no se alcanzó el ideal de una consulta por mes, establecido como adecuado para este tipo de padecimiento.

\section{Cuadro V \\ Gasto total ANUAL EN HIPERTENSIÓN ARTERIAL, PORCENTAJe del Gasto En SALUd y del PIB, SEgún ESCENARIO. MÉXICO, 1999}

$\begin{array}{ccc}\begin{array}{c}\text { Gasto total } \\ \text { anual en }\end{array} & \begin{array}{c}\text { Porcentaje del } \\ \text { gasto en salud }\end{array} & \begin{array}{c}\text { Porcentaje del } \\ \text { PIB }\end{array} \\ \text { hipertensión arterial } & 1996 & 1996 \\ \begin{array}{l}\text { Escenario promedio } \\ \$ 17953908480\end{array} & 13.95 \% & 0.71 \% \\ \text { Escenario extremo } & 51.17 \% & 2.61 \%\end{array}$

Sin embargo, al comparar el promedio de consultas por hipertenso por año en medicina familiar, resulta superior al notificado para la población en general, cifra que se ubica en 2.60. Contrario a esto, el promedio de días de hospitalización por paciente hipertenso fue inferior a la cifra para la población general (0.39 días de hospitalización por usuario). ${ }^{10}$

Para el caso concreto del uso del servicio de laboratorio, la posible explicación al elevado número de estudios de glicemia realizados se encuentra en la asociación existente entre hipertensión y diabetes mellitus como enfermedad concomitante. Pero aquí la discusión debe orientarse para aclarar si es necesario considerar este tipo de estudios como parte de la batería de exámenes utilizados por el hipertenso, considerando como ya se dijo la estrecha relación que existe entre estas dos patologías.

A diferencia de la glicemia, estudios más específicos para la evaluación del paciente hipertenso como la radiografía de tórax y el electrocardiograma, tuvieron una baja utilización que no alcanza en promedio un estudio por paciente por año. Esto podría interpretarse como una cultura no adecuada de la utilización de los servicios de salud, tanto por parte de la población hipertensa como por parte del personal de salud.

El análisis de los costos unitarios por servicio demostró una cifra baja para la consulta de medicina familiar, pero es conveniente comentar que en buena medida esto estuvo determinado por los bajos costos de los insumos variables, específicamente los medicamentos, los cuales se estimaron sobre la base de precios consolidados y por lo tanto muy por debajo de los precios de mercado. Sin embargo, la alta utilización de este servicio lo convierte en el de mayor consumo de recursos.

En contraste, a pesar de que el costo unitario del tercer nivel de atención fue el más alto, no se manifestó este predominio al hacer el análisis del costo por servicio utilizado por hipertenso, ya que, para este caso en particular, su utilización fue baja y en consecuencia el costo promedio por hipertenso también lo fue. Este mismo patrón se observa en el segundo nivel de atención.

Considerando lo anterior, e inmersos en la dinámica de la economía de la salud, si el mayor consumo de recursos por hipertenso se ubica en el primer nivel de atención, además de la identificación de las intervenciones más costo efectivas para prevenir las complicaciones propias de la enfermedad, ${ }^{11-12}$ es necesario también encontrar en el mismo sentido acciones encaminadas a la detección y control de factores de riesgo, que permitan retrasar la aparición de las manifestaciones clínicas y con ello hacer un uso más racional de los recursos destinados a la salud. 
En México, de acuerdo con lo reportado por Hernández y colaboradores, ${ }^{9}$ el gasto en salud per cápita para la población en general corresponde a 1390 pesos, cifra superior al gasto por hipertenso en el escenario promedio para la seguridad social y las instituciones del estado. Pero si se considera su alta prevalencia y el carácter crónico, entonces el atender esta necesidad de salud, obligadamente se asocia con un alto consumo de recursos, constituyéndose en un punto de atención que debe ser considerado por los sistemas de salud en la búsqueda de mecanismos que hagan más eficiente la asignación y utilización de los presupuestos destinados a la atención de la población portadora de esta patología.

Señalar que el costo promedio anual de la atención del hipertenso en la seguridad social es de 1066 pesos, puede despertar la crítica y la descalificación de lo aquí presentado; esta crítica puede acentuarse con la información correspondiente a instituciones que atienden población abierta; sin embargo, llevar esta cifra a toda la población de hipertensos, y efectuar el análisis en función del porcentaje destinado a la salud y del PIB, es alarmante. El resultado de esta comparación se torna más dramático al evaluar el escenario extremo, y con ello orientar la crítica en el sentido de señalar que no habrá presupuesto que alcance para resolver las necesidades de salud de la población. De ahí la importancia del uso racional de los recursos asignados a los sistemas de salud.

En conclusión, la hipertensión arterial, al igual que el resto de las enfermedades crónicas, es prioridad para los sistemas de salud, por lo que deberá ser objeto de estudio por equipos de salud multidisciplinarios que analicen las diferentes áreas de oportunidad en todos los niveles de atención, en la búsqueda de alternativas de manejo más eficientes.

\section{Referencias}

1. N ational Health and N utrition Examination Survey III, 1988-1991 CDS/ $\mathrm{NCHS}$.

2. Joffres MR.Awarness, treatment and control of hypertension in $C$ anada Amer JJ Hypertens 1997; 10:1097-1102.

3. Secretaría de Salud. Encuesta Nacional de Enfermedades Crónicas. Tercera Edición, México D.F.; SSA, 1996.

4. Sistema Unico de Información. Epidemiología. Sistema $\mathrm{N}$ acional de Vigilancia Epidemiológica. N úmero 52, Vol 16, Semana 52, del 26 de diciembre de 1999 al 1 de enero de 2000.

5. Calvo VC. Nuevos retos del control de la hipertensión arterial. Rev Med Instituto Mexicano del Seguro Social 1998; 36(3):199-201.

6.Villarreal Ríos E, C avazos G alván RH, G arza Elizondo ME, Guzmán Padilla JE, Montalvo A Imaguer G, Salinas Martínez AM et al. Estimación de costos en salud: una propuesta metodológica. "Modelo de presupuesto capitado" Instituto Mexicano del Seguro Social, Dirección Regional N orte, Coordinación Regional de Comunicación Social. México, D.F. 1997.

7. Secretaría de Salud. Boletín de Información Estadística. Recursos y Servicios, N 16, vol 1. Sistema N acional de Salud. México, 1996.

8.Villarreal-Ríos E, Salinas-Martínez A, G arza-Elizondo ME, N úñez-Rocha GM. Estructura del Mercado de los Servicios de Salud en México. Rev Inst Mex Seguro Soc 2000;38(5):365-369.

9. Hernández P, Zurita B, Ramírez R, Alvarez F, Cruz C. Las cuentas nacionales de salud. 1995. México, D.F.: Fundación Mexicana para la Salud (Funsalud), 1997.

10. Instituto Mexicano del Seguro Social. Compendio estadístico anual de oferta y demanda, servicios médicos otorgados y morbi-mortalidad en N uevo León. Coordinación D elegacional de Informática Médica. Jefatura Delegacional de Prestaciones Médicas. D elegación Regional N uevo León. Instituto Mexicano del Seguro Social. Monterrey, N .L., 1998.

11. McMurray J.The health economics of the treatment of hyperlipidemia and hypertension. Am J Hypertens 1999;(12): 99-104.

12. Shepard D, Hodgkin D. Cost effectiveness of intensive treatment of hypertension. AM J Manag Care 1998: 765-769. 